DR REINHART SPEECKAERT (Orcid ID : 0000-0002-9421-3546)

PROFESSOR JORIS R DELANGHE (Orcid ID : 0000-0002-5702-6792)

Article type : Original Article

\title{
Infrared spectroscopy as a novel tool to diagnose onychomycosis
}

Short title: Infrared spectroscopy and onychomycosis

S. De Bruyne ${ }^{1,2}$, R. Speeckaert ${ }^{3}$, J. Boelens ${ }^{1,2}$, M.-P. Hayette ${ }^{4}$, M. Speeckaert ${ }^{5}$ and J. Delanghe ${ }^{1,2}$

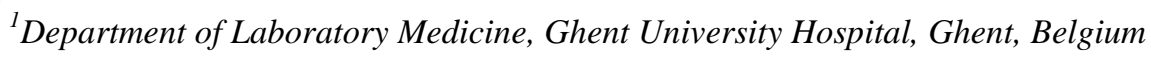

${ }^{2}$ Department of Clinical Chemistry, Microbiology and Immunology, Ghent University, Ghent, Belgium

${ }^{3}$ Department of Dermatology, Ghent University Hospital, Ghent, Belgium

${ }^{4}$ Department of Clinical Microbiology, CHU Sart-Tilman, Liege, Belgium

${ }^{5}$ Department of Nephrology, Ghent University Hospital, Ghent, Belgium

Corresponding author: Prof. Dr. Joris Delanghe

E-mail: Joris.Delanghe@UGent.be

This article has been accepted for publication and undergone full peer review but has not been through the copyediting, typesetting, pagination and proofreading process, which may lead to differences between this version and the Version of Record. Please cite this article as doi: 10.1111/bjd.17199

This article is protected by copyright. All rights reserved. 
List of abbreviations: ATCC, American Type Culture Collection; ATR-FTIR, Attenuated total reflectance Fourier transform infrared spectroscopy; CW, Calcofluor white; HCA, Hierarchical cluster analysis; ITS, Internal transcribed spacer; MALDI-TOF MS, Matrix-assisted laser desorption/ionization time-of-flight mass spectroscopy; MIR, Mid-infrared; PAS, Periodic-acidSchiff; PCR, Polymerase chain reaction; KOH, Potassium hydroxide; PCA, Principal component analysis; SDA, Sabouraud dextrose agar; SIMCA, Soft independent Modeling of Class Analogy; SNV, Standard normal variate; UK NEQAS, United Kingdom National External Quality Assessment Service

Keywords: Fourier transform infrared spectroscopy; medical laboratory science; mid-infrared absorbance spectroscopy; onychomycosis; vibrational spectroscopy

Conflict of Interest Disclosures: None reported.

Funding/Support: None reported.

Acknowledgement: We are indebted to Tessa Moreels, Jochen Van Herck, Joris Van Caenegem, Els De Grauwe and Saskia Verhofstede for providing assistance in the spectral analysis and the identification of clinical isolates, and Prof. dr. Thomas De Beer and Dr. Tinne Monteyne for the helpful discussions concerning infrared spectroscopy and chemometrics.

\section{What's already known about this topic?}

- The determination of causative organisms of onychomycosis is still not optimal.

- There remains a need for a cheap, fast and easy-to-perform diagnostic tool with highly distinctive capacities.

This article is protected by copyright. All rights reserved. 


\section{What does this study add?}

- Attenuated total reflectance Fourier transform infrared spectroscopy (ATR-FTIR) is a potential novel tool in the detection and differentiation of causative organisms of onychomycosis.

\section{ABSTRACT}

Background: The determination of causative organisms of onychomycosis is still not optimal. There remains a need for a cheap, fast and easy-to-perform diagnostic tool with highly distinctive capacities.

Objectives: To determine whether ATR-FTIR spectroscopy can detect and differentiate causative agents in a culture based, ex vivo nail and in vivo nail model.

Methods: A methodological study was conducted. Both the ex vivo nail model and in vivo pilot study were carried out in an academic university hospital.

Results: Analysis of cultured fungi revealed spectral differences for dermatophytes (16921606 and 1044-1004 $\left.\mathrm{cm}^{-1}\right)$ and non-dermatophytes/yeasts $\left(973-937 \mathrm{~cm}^{-1}\right)$, confirmed by dendrograms showing an excellent separation between samples from different genera or species. Exploration of dermatophytes, non-dermatophytes and yeasts growing on ex vivo nails exposed prominent differences from 1200 to $900 \mathrm{~cm}^{-1}$. Prediction models resulted in a 
96.9\% accurate classification of uninfected nails and nails infected with dermatophytes, nondermatophytes and yeasts. Overall correct classification rates of $91.0 \%, 97.7 \%$ and $98.6 \%$ were obtained for discrimination between dermatophyte, non-dermatophyte and yeast genera or species, respectively. Spectra of in vivo infected and uninfected nails also revealed distinct spectral differences $\left(3000-2811 \mathrm{~cm}^{-1}, 1043-950 \mathrm{~cm}^{-1}\right.$ and $\left.1676-1553 \mathrm{~cm}^{-1}\right)$, illustrated by two main clusters (uninfected versus infected) on a dendrogram.

Conclusions: Our data suggest that ATR-FTIR spectroscopy may be a promising, fast and accurate method to determine onychomycosis, including identification of the causative organism, bypassing the need for lengthy fungal cultures.

\section{INTRODUCTION}

Onychomycosis refers to nail infections caused by fungi such as dermatophytes (tinea unguium), nondermatophytes and yeasts ${ }^{1,2}$. During the last decades, the rate of onychomycosis is globally rising in relation to increases of important risk factors (e.g. diabetes mellitus) and lifestyle changes (e.g. occlusive footwear) ${ }^{1,3,4}$. Despite the fact that onychomycosis may be strongly suspected based upon physical examination, laboratory evidence is needed in order to initiate appropriate treatment for several reasons ${ }^{5}$. Onychomycosis has variable clinical presentations and may appear similar to other onychopathies (e.g nail psoriasis) ${ }^{5,6}$. Furthermore, the efficacy of available drugs differs between dermatophyte or yeast/non-dermatophyte onychomycosis ${ }^{7}$. At present, typically applied laboratory tests for the diagnosis of onychomycosis include direct microscopy with potassium hydroxide $(\mathrm{KOH})$ and fungal culture or histopathological examination of periodic-acid-Schiff (PAS)-stained nail clippings and fungal culture. However, this approach is hampered by several shortcomings: direct microscopic examination lacks specificity and is operator dependent, whereas in vitro culture suffers

This article is protected by copyright. All rights reserved. 
from weak sensitivity and false negative results ${ }^{5}$. In addition, fungal culture is time consuming and requires up to 2-6 weeks for the results ${ }^{5-7}$. Calcofluor white $(\mathrm{CW})$ examination, polymerase chain reaction (PCR), in vivo confocal microscopy, optical coherence tomography, nail digital onychoscopy and matrix-assisted laser desorption/ionization time-of-flight mass spectroscopy (MALDI-TOF MS) have emerged as additional diagnostic methods ${ }^{8-13}$. Nonetheless, detection and differentiation of causative organisms of onychomycosis is still not ideal and there remains a need for a cheap, fast and easy-to-perform alternative technique with highly distinctive capacities. Attenuated total reflectance Fourier transform infrared spectroscopy (ATR-FTIR) is an absorptive method that depends upon changes in permanent dipole moments during excitation in the mid-infrared (MIR) region (4000-400 $\mathrm{cm}^{-1}$ ) and fits all the characteristics for the development of a new tool to diagnose onychomycosis. It is a non-destructive, label-free and specific technique that can provide complete information on the chemical composition of biological samples ${ }^{14}$. In this technique, a beam of radiation penetrates a fragment of a wavelength beyond the reflecting surface and subsequently loses energy at the wavelength where the sample absorbs. The resultant attenuated total reflection is detected and plotted as a function of wavelength which gives rise to the characteristic absorption spectrum ${ }^{15}$. Biomedical applications of FTIR spectroscopy involve the identification and typing of several microorganisms such as Candida ${ }^{16}$ and Trichophyton ${ }^{17}$ species. Furthermore, IR analysis of nails has been studied as an indicator of health by several research groups ${ }^{18-20}$. The present study explores the possibilities of ATR-FTIR spectroscopy in the detection and differentiation of causative agents of onychomycosis by (1) analysing the spectral properties of causative microorganisms plated out on Sabouraud dextrose agars (SDAs), (2) analysing the spectral properties of nails infected with several causative agents in an ex vivo model, and (3) analysing the spectral properties of nails obtained from clinical routine.

This article is protected by copyright. All rights reserved. 


\section{MATERIALS AND METHODS}

\section{Fungal strains}

Causative microorganisms of onychomycosis, identified with conventional phenotypic methods $(n=20)$, were collected by the Department of Medical Microbiology of the University Hospital of Ghent (Belgium). Strains, confirmed by internal transcribed spacer (ITS) sequencing ( $n=7)$, were provided by the National Reference Center for Mycosis at the University Hospital of Liège (Belgium). Furthermore, some strains were derived from the United Kingdom National External Quality Assesment Service (UK NEQAS, n=2) and the American Type Culture Collection (ATCC, n=3). All strains were cultured on SDA plates (Sigma Aldrich Chemie GmbH, Schnelldorf, Germany) and incubated at $28^{\circ} \mathrm{C}$ (Table 1). After one week incubation, fungi were harvest by carefully scraping the agar plate with a surgical knife at 5 different locations. Mean spectra were used for further data analysis.

\section{Collection and ex vivo infected nails}

Distal fingernail $(\mathrm{n}=158)$ and toenail $(\mathrm{n}=159)$ clippings were obtained from 73 adult volunteers (36 males and 37 females, age: $21.0 \pm 4.8$ years (median \pm standard deviation)) without suspected nail abnormalities based upon physical examination. The presence of nail polish was an exclusion criterion. Nail fragments were removed from visible dirt with a pincette, cut to $2 \times 2 \mathrm{~mm}$ pieces, and subsequently cleaned in an ultrasonic water bath for $30 \mathrm{~min}$ in $77 \%$ ethanol (Dax Alcoliquid 77, Dialex Biomedica, Sweden). Subsequently, fragments were air-dried and stored in sterile Petri dishes until analysis or inoculation. During the inoculation process, ventral surfaces of 10 fingernail and 10 toenail fragments were placed directly onto SDA plates inoculated with dermatophytes, nondermatophytes and yeasts to allow penetration through the intercellular spaces ${ }^{21}$. After 2 weeks incubation at $28^{\circ} \mathrm{C}$, nails were removed from culture and dried in a vacuum desiccator $\left(24 \mathrm{~h}, 20^{\circ} \mathrm{C}\right)$. After drying, ventral surfaces were scraped with a surgical knife to remove adherent fungus and

This article is protected by copyright. All rights reserved. 
thereby allowing the measurement of invaded fungi, exclusively. Infection of nail fragments was observed macroscopically and microscopically, following CW stain (Fungi-Fluor ${ }^{\circledR}$ Stain Solution, Polysciences Inc., Pennsylvania, USA). CW is a non-specific fluorochrom that binds to cellulose and chitin contained in the cell walls of fungi and other cellulose-containing organisms. Ventral nail scrapings were placed onto a clean glass slide and one drop of Fungi-Fluor ${ }^{\circledR}$ Staining Solution, a $0.05 \%$ solution of high purity Cellufluor in deionized water with potassium hydroxide added as a clearing agent, was applied directly to the specimen. Subsequently, samples were evaluated for the presence of yeasts and fungi using a microscope equipped with fluorescence contrast (Leica DM2000, Leica Microsystems, Germany).

\section{Pilot model: in vivo nails}

Nineteen patients with suspected distal toenail onychomycosis (12 males and 7 females, age: $57.0 \pm$ 12.2 years) were recruited from the Department of Dermatology of the University Hospital of Ghent, Belgium. Nail specimens were obtained with standard nail clippers and identified in routine fashion by $\mathrm{CW}$ stain and culture. Cultures were checked periodically for growth during a minimum of 4 weeks. Furthermore, 11 healthy volunteers were included ( 9 males and 2 females, age: $58.0 \pm 21.0$ years). For analysis, nail samples were cleaned in an ultrasonic water bath for $15 \mathrm{~min}$ in distilled water to remove surface contaminants. Immediately after the wash, nail samples were dried with a heated laboratory dryer $(5 \mathrm{~min})$ and powdered $(30 \mathrm{~Hz}, 10 \mathrm{~min})$ using a ball mill with $5 \mathrm{~mm}$ diameter

stainless-steel balls (MM400, Retsch, Germany) to ensure sample homogeneity. The study was approved by the Ethics Committee of Ghent University Hospital (EC UZG 2016/1250).

This article is protected by copyright. All rights reserved. 


\section{ATR-FTIR spectroscopic analysis}

FTIR spectra of cultured fungi, intact nail fragments (ventral surfaces) and nail powders were obtained using a Perkin Elmer Spectrum Two ATR-FTIR spectrometer accommodated with the universal ATR Accessory (ZnSe crystal) and Spectrum 10 software (Perkin Elmer, Waltham, MA, USA) in the range from $4000 \mathrm{~cm}^{-1}$ to $450 \mathrm{~cm}^{-1}$ at a spectral resolution of 4 $\mathrm{cm}^{-1}$ (5 co-added scans), and subsequently incorporated into SIMCA version 14.1 (MKS Data Analytics Solutions) software in order to preprocess and analyze the acquired spectral data. Figure 1a shows the mean raw absorbance spectra from cultured Trichophyton rubrum (T. rubrum) strains, intact control toenails and intact toenails infected ex vivo with T. rubrum. The mean absorbance spectra of powdered control toenails and powdered toenails infected in vivo with T. rubrum can be found in Figure $1 \mathrm{~b}$.

\section{Multivariate data analysis}

As mentioned above, all samples were scanned in the MIR region from $4000 \mathrm{~cm}^{-1}$ to $450 \mathrm{~cm}^{-}$ ${ }^{1}$, but narrower regions which contained the most valuable spectral information were chosen for multivariate data analysis. To remove irrelevant scatter light and standardize the spectroscopic signals, several spectral filters were employed. Spectral data from the culture based and ex vivo nail model were mean-centered, converted to their second derivatives with a Savitsky-Golay algorithm (15 smoothing points), normalized using the standard normal variate (SNV) method and statistically analyzed by unsupervised pattern recognition methods, such as principal component analysis (PCA) and hierarchical cluster analysis (HCA), and supervised pattern recognition methods such as soft independent modeling of class analogy (SIMCA). In case of the in vivo model, spectra were converted to their first 
derivative and normalized using SNV before chemometric analysis. The advantages of derivative techniques are twofold. In first derivative, resolution is enhanced since the rate of change of absorbance (A) with respect to wavelength $(\lambda)$ is examined $(\mathrm{d} A / \mathrm{d} \lambda)$. The second derivative measures alterations in the rate of change of absorbance $\left(\mathrm{d}^{2} \mathrm{~A} / \mathrm{d} \lambda^{2}\right)$ and results in a negative peak for each band and shoulder in the absorption spectrum. For more complex spectra (e.g. biological spectra), differentiation is utile to resolve and locate overlapping bands. Sharp bands are intensified at the expense of broader ones, which may allow for the selection of suitable peaks, even when broad bands are beneath ${ }^{15}$.

\section{Principal component analysis (PCA)}

PCA is a multivariate projection method designed to reduce dimensionality of data from hundreds of spectral data points into a few orthogonal principal components (PCs) such that an overview of the data is obtained ${ }^{22,23}$. The first $\mathrm{PC}$ is the line in the K-dimensional space, that best approximates the data in the least squares sense and goes through the average point. Most of the time, one PC is insufficient to model the systematic variation of a data set and a second PC is calculated. The second PC is orthogonal to the first PC, passes through the average point and improves the approximation of the X-data as much as possible. When two PCs have been derived, they together define a plane. By projecting all the observations onto this low-dimensional sub-space and plotting the results, it is possible to visualize the structure of the investigated data $\operatorname{set}^{23}$. The coordinates of the observations on this plane are called scores, and the plotting of such a projected configuration is known as a score plot $^{23}$. Frequently, one or two PCs are not enough to adequately summarize the information in a data set and more PCs are calculated ${ }^{23}$. In this work, PCA was used to illustrate differences 
between all of the studied material and to explore whether this technique can result in a clustering of samples, which is useful for the creation of classification models.

\section{Hierarchical cluster analysis (HCA)}

HCA was used to graphically display spectral similarities in selected windows as a dendrogram, which shows clustering in two dimensions by graphical means ${ }^{24}$. Similarity is expressed as heterogeneity, where an increase in heterogeneity corresponds to an increase in dissimilarity ${ }^{24}$. Euclidean distance was used to calculate spectral similarities and the Ward hierarchical agglomerative method was used to establish clusters. In this algorithm, homogeneous objects are clustered as much as possible by combining the spectra that form the smallest variance distance ${ }^{24}$. The vertical axis of the dendrogram indicates the loss in within cluster similarity (i.e. the variance increase), when clusters are merged. The scale on the vertical axis is rather arbitrary.

\section{Soft independent modeling of class analogy (SIMCA)}

With relevant wavenumber variables identified, we proceeded to evaluate the predictive properties of the ex vivo nail model by using SIMCA. The complete dataset was divided into a training and test set. Samples were randomly assigned to the training set, consisting of about $65 \%$ of the samples, and the test set, composed of the remaining $35 \%$ of the samples. The same procedure was repeated three times with different compositions of both sets to ensure the inclusion of all samples in the test set at least once. SIMCA is a supervised classification method that models each class of samples separately by defining a number of

This article is protected by copyright. All rights reserved. 
PCs derived from PCA. First of all, SIMCA defines the optimal number of PCs required to describe each training class individually by using a cross-validation procedure. Secondly, classification rules are set-up by taking two critical values into account: (1) the Euclidean distances (= orthogonal distances) towards the model and (2) the Mahalanobis distances calculated in the space of the scores. Those distances define a limited space around the samples in one specific class and form the boundaries of the model. If unknown (test set) samples are situated within the limited space of a training class, then the samples are assigned to that specific class ${ }^{22}$.

\section{RESULTS}

\section{Cultured microorganisms}

In total, 32 different fungal microorganisms were investigated, including 20 dermatophytes, 8 nondermatophytes and 4 yeasts (Table 1). In second derivative of spectral ranges, the regions between $1692-1606 \mathrm{~cm}^{-1}$ (amide I band containing mainly $\mathrm{C}=\mathrm{O}$ stretching vibrations of protein peptide bonds) and 1044-1004 $\mathrm{cm}^{-1}$ (part of the carbohydrate region ranging from 1200 till $900 \mathrm{~cm}^{-1}$ ) showed clear differences among the different dermatophytes. Based on similarity and clustering, the dendrogram based on those spectral ranges contained two main clusters representing Microsporum spp. (cluster 1) and Trichophyton spp. (cluster 2, Figure 2a). In the case of non-dermatophytes and yeasts, important spectral differences were noticed between $973-937 \mathrm{~cm}^{-1}$ (part of the carbohydrate region). The resulting dendrogram showed clustering of all Candida species and non-dermatophyte fungi (Fusarium spp., Penicillium spp. and Acremonium spp.), with exception of Scopulariopsis brevicaulis that formed a separate cluster (Figure 2b).

This article is protected by copyright. All rights reserved. 


\section{Ex vivo nail model}

PCA was applied in order to evaluate spectral differences between uninfected nails $(n=200)$ and nails infected with causative agents of onychomycosis $(n=434)$. Most prominent spectral differences were found in the range from 1200 to $900 \mathrm{~cm}^{-1}$ (carbohydrate region, Figure 3a). The resulting score plot showed a clear distinction between uninfected and infected nails (Figure 3b). SIMCA was applied to test whether appropriate classification models, based on the $1200-900 \mathrm{~cm}^{-1}$ spectral range, could be obtained which also might serve to predict unknown samples. A first classification model $\left(\mathrm{M}_{1}\right)$ was designed to differentiate between uninfected nails and nails infected with dermatophytes, nondermatophytes and yeasts. The obtained model generated a correct classification ratio of $96.9 \%$ since only 7 samples out of 214 test samples were misclassified. Since a good model was acquired, it was verified whether this model could be extended to discriminate between different dermatophyte, nondermatophyte and yeast species. A second classification model $\left(\mathrm{M}_{2}\right)$ was developed to discriminate between nails infected with different dermatophyte species. The external validation resulted in a correct classification rate of $91.0 \%$ since the test set showed only 7 misclassified samples out of 238 nails infected with dermatophytes. Furthermore, a third $\left(\mathrm{M}_{3}\right)$ and fourth classification model $\left(\mathrm{M}_{4}\right)$ were designed to differentiate between nails infected with non-dermatophytes and yeasts, respectively. The obtained models showed a correct classification ratio of $97.7 \%$ and $98.6 \%$, respectively (Table 2).

\section{Pilot model: In vivo nails}

A proof of concept was conducted using a pilot in vivo nail model. Demographic characteristics, comorbidities and medication use of subjects enrolled in the in vivo model can be found in Table 3 . From the 19 toenails with clinical suspicion of distal toenail onychomycosis, 7 samples were negative for both $\mathrm{CW}$ stain and culture. Twelve samples were positive for $\mathrm{CW}$ and/or culture. From these samples, 7 were identified as T. rubrum and 2 as Candida parapsilosis. Three samples showed 
mycelia on CW without fungal growth. Toenails with negative $\mathrm{CW}$ and culture, together with 11 nails from healthy volunteers were grouped as uninfected. Nails positive for CW stain and/or culture were classified as infected. In first derivative, distinct spectral differences were found in the $1043-950 \mathrm{~cm}^{-1}$ region (part of the carbohydrate region). In addition, spectral differences were found in the 3000-2811 $\mathrm{cm}^{-1}$ region, a region assigned to (a)symmetric $\mathrm{CH}_{2}$ and $\mathrm{CH}_{3}$ stretching vibrations of lipids, and the $1676-1553 \mathrm{~cm}^{-1}$ region (amide I band, Figure 4a). PCA performed on these regions revealed clear clustering of uninfected and infected nails in the score plot (Figure 4b). Furthermore, HCA resulted in a dendrogram with two main clusters representing infected (cluster 1) and uninfected nails (cluster 2, Figure 4c). One sample with negative CW/culture was situated within cluster 1 . Notice the high level of similarity (i.e. low dissimilarity value) between the two nails infected with C. parapsilosis.

\section{DISCUSSION}

In the presence of predisposing factors, the nail plate offers an ideal ecological niche for fungal organisms, where they can persist for a long time due to a lack of effective cellmediated immunity ${ }^{25}$. The structure and biosynthesis of fungal cell walls are unique to the fungi, and therefore well suited for biochemical characterization. The fungal cell wall has been shown to be mainly composed of glycoproteins and polysaccharides. In addition, minor cell wall components are present, which vary amongst species of fungi ${ }^{26}$.

Analysis of cultured dermatophytes revealed obvious spectral differences in the range from 1692 to $1606 \mathrm{~cm}^{-1}$ between different species. This region, the so-called amide I band, contains mainly $\mathrm{C}=\mathrm{O}$ stretching vibrations of peptide bands and is therefore used as a proxy for proteins and their secondary structure ${ }^{27}$. Furthermore, distinct spectral differences were found between $1044-1004 \mathrm{~cm}^{-1}$ for the dermatophytes and between $973-937 \mathrm{~cm}^{-1}$ for the non- 
dermatophytes and yeasts. Carbohydrates, whether mono- or polymeric, are known to evoke strong bands in the $1200-900 \mathrm{~cm}^{-1}$ region due to ring C-O-C vibrations ${ }^{27}$. As a consequence, spectral differences in those regions can be assigned to the existence of characteristic differences in the protein and polysaccharide content of the fungal microorganisms. As an example, it has been reported that $T$. rubrum shows differences in the galactomannan structure ( $\alpha$-mannopyranose, galactofuranose and mannofuranose attached to a peptide backbone), compared to other Trichophyton species ${ }^{7}$.

In our ex vivo nail model, we have found that the region with the best discriminating features was localized in the carbohydrate region. Prediction models based on this region showed overall excellent accuracy values, even on species level. These results show that FTIR spectroscopic examination of the polysaccharide content could be a reliable technique for the detection and differentiation of causative organisms of onychomycosis. These findings were confirmed, to a certain extent, in the analysis of in vivo toenail samples. However, it is important to be aware of some important differences between the ex vivo and in vivo model. In the ex vivo model, nails were washed with ethanol before incubation with cultured microorganisms to avoid contamination of the agars, while in the in vivo model nails were washed with distilled water. As ethanol is a polar solvent, additional effects such as extraction of surface lipids can be expected ${ }^{28}$. Another significant difference is the analysis of intact versus powdered nails. In the in vivo model, powdering of nails was necessary to ensure sample homogeneity since macroscopic changes (e.g. thickening of the nail, irregular surfaces) enhanced spectral noise. For the in vivo model, discriminating differences between onychomycotic and uninfected nails were found in the first derivative of the polysaccharide region and amide I band. In addition, significant spectral differences were observed in the spectral range from 3000 to $2811 \mathrm{~cm}^{-}$ ${ }^{1}$, a region assigned to (a)symmetric $\mathrm{CH}_{2}$ and $\mathrm{CH}_{3}$ stretching vibrations of lipids. Lipids play an essential role in fungal physiological processes associated with growth, sporulation and germination ${ }^{7}$. Lipid compositions have been reported to vary between different dermatophytic strains, especially in

This article is protected by copyright. All rights reserved. 
the case of T. rubrum $^{29}$. However it has to be noted that lipid profiles depend on the fungal growth stage as well ${ }^{30}$.

The present study reports for the first time differences in FTIR spectra between uninfected nails and nails infected with causative organisms of onychomycosis. Based on our findings, it can be stated that ATR-FTIR spectroscopy could be a promising technique for the diagnosis of onychomycosis for several reasons. The proposed technique presents attractive features such as being fast, simple, non-destructive and even portable to work on-field ${ }^{31}$. Furthermore, a minimum level of expertise is required with relatively low setup costs, i.e. one-time fixed cost for the ATR-FTIR spectrometer. On the other hand, the value of confirmatory testing before initiation of therapy for onychomycosis is currently under discussion. It has been stated that confirmatory diagnostic testing before initiating systemic therapy for clinically diagnosed onychomycosis is more expensive than initiating treatment in all suspected cases ${ }^{32}$. FTIR spectroscopy has the potential to significantly reduce delays in diagnosis and treatment in a very cost-effective way, since the technique does not require any reagents or extensive sample handlings, which is in marked contrast with other diagnostic tools such as molecular techniques ${ }^{31}$. Furthermore, the technique presents other interesting opportunities. FTIR spectra of nails represent a mine of information. The collection of this kind of information might be employed for providing a posteriori diagnostic analysis ${ }^{31}$ in other applications such as the diagnosis of psoriasis ${ }^{20}$ and diabetes mellitus ${ }^{18}$. In psoriatic nails, specific molecular changes due to degradation mechanisms were observed in the secondary structure of keratin by studying the amide I region. In addition, absorption bands at 1170 and $1040 \mathrm{~cm}^{-1}$, which were assigned to $\mathrm{S}-\mathrm{O}$ bonds of the cysteic acid unit, were reported ${ }^{20}$. The different biochemical fingerprint of psoriatic and onychomycotic nails could be used a differential diagnostic tool.

This article is protected by copyright. All rights reserved. 
However, the proposed new diagnostic technique can be faced with some challenges. FTIR measurements of onychomycosis may also detect spectral characteristics of contaminating bacteria $^{7}$, which can bee seen in conditions such as the green nail syndrome caused by Pseudomonas aeruginosa ${ }^{33}$. Nevertheless, since bacteria and fungi have a completely different cellular makeup, it is rather unlikely that this will lead to false-negative and falsepositive results. Furthermore, spectral properties of antifungal medication might provoke significant interference. In these situations, samples may remain unclassified. Therefore, fungal culture might occasionally still be necessary for identification. At last, the need for large databases of reference spectra that sufficiently include most of the variation in nail matrices and phenotypic heterogeneity of causative microorganisms is expected to be the biggest hurdle.

All together, it can be concluded that causative organisms of onychomycosis can be detected and differentiated in an easy and fast way by means of ATR-FTIR spectroscopy. Large-scale investigations on clinical onychomycosis diagnostics are necessary to determine its definite position as a potential new tool for the diagnosis of onychomycosis compared to currently employed techniques.

\section{REFERENCES}

1. Rosen T, Friedlander SF, Kircik L, et al. Onychomycosis: epidemiology, diagnosis, and treatment in a changing landscape. J Drugs Dermatol. 2015;14:223-33.

2. Gupta AK, Jain HC, Lynde CW, et al. Prevalence and epidemiology of onychomycosis in patients visiting physicians' offices: A multicenter Canadian survey of 15,000 patients. J Am

This article is protected by copyright. All rights reserved. 
Acad Dermatol. 2000;43:244-8.

3. Tchernev G, Penev PK, Nenoff P, et al. Onychomycosis: Modern diagnostic and treatment approaches. Vol. 163, Wiener Medizinische Wochenschrift. 2013. p. 1-12.

4. Papini M, Piraccini BM, Difonzo E, et al. Epidemiology of onychomycosis in Italy: Prevalence data and risk factor identification. Mycoses. 2015;58:659-64.

5. Petinataud D, Berger S, Ferdynus C, et al. Optimising the diagnostic strategy for onychomycosis from sample collection to FUNGAL identification evaluation of a diagnostic kit for real-time PCR. Mycoses. 2016;59:304-11.

6. Jung MY, Shim JH, Lee JH, et al. Comparison of diagnostic methods for onychomycosis, and proposal of a diagnostic algorithm. Clin Exp Dermatol. 2015;40:479-84.

7. Smijs TG, Jachtenberg JW, Pavel S, et al. Detection and differentiation of causative organisms of onychomycosis in an ex vivo nail model by means of Raman spectroscopy. J Eur Acad Dermatology Venereol. 2014;28:1492-9.

8. Weinberg JM, Koestenblatt EK, Tutrone WD, et al. Comparison of diagnostic methods in the evaluation of onychomycosis. J Am Acad Dermatol. 2003;49:193-7.

9. Brillowska-Dabrowska A, Swierkowska A, Lindhardt Saunte DM, et al. Diagnostic PCR tests for Microsporum audouinii, M. canis and Trichophyton infections. Med Mycol. 2010;48:48690.

10. Rothmund G, Sattler EC, Kaestle R, et al. Confocal laser scanning microscopy as a new valuable tool in the diagnosis of onychomycosis - comparison of six diagnostic methods. Mycoses. 2013;56:47-55.

11. Abuzahra F, Spöler F, Först M, et al. Pilot study: Optical coherence tomography as a noninvasive diagnostic perspective for real time visualisation of onychomycosis. Mycoses. 2010;53:334-9.

12. Piraccini BM, Balestri R, Starace M, et al. Nail digital dermoscopy (Onychoscopy) in the diagnosis of onychomycosis. J Eur Acad Dermatology Venereol. 2013;27:509-13.

This article is protected by copyright. All rights reserved. 
13. Pföhler C, Hollemeyer K, Heinzle E, et al. Matrix-assisted laser desorption/ionization time-offlight mass spectrometry: A new tool in diagnostic investigation of nail disorders? Exp Dermatol. 2009;18:880-2.

14. Stuart BH. Infrared spectroscopy: fundamentals and applications. Chichester (UK): Wiley; 2004.

15. Zelig U, Barlev E, Bar O, et al. Early detection of breast cancer using total biochemical analysis of peripheral blood components: a preliminary study. BMC Cancer. 2015;15:408.

16. Toubas D, Essendoubi M, Adt I, et al. FTIR spectroscopy in medical mycology: Applications to the differentiation and typing of Candida. Anal Bioanal Chem. 2007;387:1729-37.

17. Ergin Ç, Ilkit M, Gök Y, et al. Fourier transform infrared spectral evaluation for the differentiation of clinically relevant Trichophyton species. J Microbiol Methods. 2013;93:21823.

18. Coopman R, Van de Vyver T, Kishabongo AS, et al. Glycation in human fingernail clippings using ATR-FTIR spectrometry, a new marker for the diagnosis and monitoring of diabetes mellitus. Clin Biochem. 2017;50:62-7.

19. Sakudo A, Kuratsune H, Kato YH, et al. Secondary structural changes of proteins in fingernails of chronic fatigue syndrome patients from Fourier-transform infrared spectra. Clin Chim Acta. 2009;402:75-8.

20. Coroaba A, Pinteala T, Chiriac A, et al. Degradation Mechanism Induced by Psoriasis in Human Fingernails: A Different Approach. J Invest Dermatol. 2016;136:311-3.

21. Bulgheroni A, Frisenda L, Subissi A, et al. A Hydroxypropyl Chitosan (HPCH) Based Medical Device Prevents Fungal Infections: Evidences from an In Vitro Human Nail Model. Open Dermatol J. 2015;9:4-9.

22. Custers D, Cauwenbergh T, Bothy JL, et al. ATR-FTIR spectroscopy and chemometrics: An interesting tool to discriminate and characterize counterfeit medicines. J Pharm Biomed Anal. 2015;112:181-9.

This article is protected by copyright. All rights reserved. 
23. Eriksson L, Johansson E, Kettaneh-Wold N, et al. PCA. Multi- Megavariate Data Anal Part 1, Basic Princ Appl. 2006;39-62.

24. Kucuk Baloglu F, Baloglu O, Heise S, Bet al. Triglyceride dependent differentiation of obesity in adipose tissues by FTIR spectroscopy coupled with chemometrics. J Biophotonics. 2017;10:1345-55.

25. Grover C, Khurana A. Onychomycosis: Newer insights in pathogenesis and diagnosis. Indian J Dermatology, Venereol Leprol. 2012;78:263.

26. Bowman SM, Free SJ. The structure and synthesis of the fungal cell wall. BioEssays. 2006;28:799-808.

27. Kaminskyj S, Jilkine K, Szeghalmi A, et al. High spatial resolution analysis of fungal cell biochemistry - Bridging the analytical gap using synchrotron FTIR spectromicroscopy. FEMS Microbiol Lett. 2008;284:1-8.

28. Gniadecka M, Faurskov Nielsen O, Christensen DH, et al. Structure of water, proteins, and lipids in intact human skin, hair, and nail. J Invest Dermatol. 1998;110:393-8.

29. Khuller GK, Sadhna S, Deo D. Dermatophyte lipids-Composition and regulation of phospholipids. Indian J Clin Biochem. 2000;15:51-9.

30. Swanson R, Stock JJ. Biochemical Alterations of Dermatophytes during Growth. Appl Microbiol. 1966;14:438-44.

31. De Bruyne S, Speeckaert MM, Delanghe JR. Applications of mid-infrared spectroscopy in the clinical laboratory setting. Crit Rev Clin Lab Sci. 2018;55:1-20.

32. Mikailov A, Cohen J, Joyce C, et al. Cost-effectiveness of confirmatory testing before treatment of onychomycosis. JAMA Dermatology. 2016;152:276-81.

33. Chiriac A, Brzezinski P, Foia L, et al. Chloronychia: Green nail syndrome caused by Pseudomonas aeruginosa in elderly persons. Clin Interv Aging. 2014;10:265-7.

This article is protected by copyright. All rights reserved. 


\section{TABLES}

Table 1: Overview of the group, genus, species and type of identification of the different fungal microorganisms included in the study.

Strains used in the ex vivo nail model are indicated ' + '.

\begin{tabular}{|c|c|c|c|c|c|}
\hline Group & Genus & Species & Identification & Ex vivo nail model & $\mathbf{N}^{\circ}$ \\
\hline \multirow[t]{20}{*}{ Dermatophytes } & \multirow[t]{16}{*}{ Trichophyton } & erinacei & UKNEQAS & - & 1 \\
\hline & & interdigitale & UKNEQAS & - & 2 \\
\hline & & interdigitale & ITS sequencing & + & 3 \\
\hline & & mentagrophytes & phenotypic & + & 4 \\
\hline & & mentagrophytes & phenotypic & - & 5 \\
\hline & & mentagrophytes & phenotypic & - & 6 \\
\hline & & rubrum & phenotypic & + & 7 \\
\hline & & rubrum & phenotypic & + & 8 \\
\hline & & rubrum & phenotypic & + & 9 \\
\hline & & rubrum & phenotypic & - & 10 \\
\hline & & rubrum & phenotypic & - & 11 \\
\hline & & rubrum & phenotypic & - & 12 \\
\hline & & soudanense & ITS sequencing & + & 13 \\
\hline & & tonsurans & phenotypic & & 14 \\
\hline & & tonsurans & ITS sequencing & + & 15 \\
\hline & & violaceum & ITS sequencing & + & 16 \\
\hline & \multirow[t]{3}{*}{ Microsporum } & canis & phenotypic & - & 17 \\
\hline & & canis & ITS sequencing & + & 18 \\
\hline & & persicolor & ITS sequencing & + & 19 \\
\hline & Arthroderma & benhamiae & ITS sequencing & + & 20 \\
\hline \multirow[t]{8}{*}{ Non-dermatophytes } & Acremonium & - & phenotypic & + & 21 \\
\hline & Acremonium & - & phenotypic & + & 22 \\
\hline & Acremonium & - & phenotypic & + & 23 \\
\hline & Fusarium & - & phenotypic & + & 24 \\
\hline & Fusarium & - & phenotypic & + & 25 \\
\hline & Penicillium & - & phenotypic & + & 26 \\
\hline & Penicillium & - & phenotypic & + & 27 \\
\hline & Scopulariopsis & brevicaulis & phenotypic & + & 28 \\
\hline \multirow[t]{4}{*}{ Yeasts } & \multirow[t]{4}{*}{ Candida } & albicans & ATCC 24433 & + & 29 \\
\hline & & parapsilosis & phenotypic & - & 30 \\
\hline & & parapsilosis & ATCC 22019 & + & 31 \\
\hline & & krusei & ATCC 6258 & + & 32 \\
\hline
\end{tabular}

Abbreviations: ATCC, American Type Culture Collection; ID, identification; ITS, internal transcribed spacer; $\mathrm{N}^{\circ}$, number; UKNEQAS, United Kingdom National External Quality Assesment Service. 
Table 2: Overview of the classification system to discriminate between uninfected nails and nails infected with causative microorganisms. The number of samples infected ex vivo with different causative microorganisms, samples included in the training set, samples included in the test set and the classification accuracy of test set samples are illustrated. Classification model $1\left(\mathrm{M}_{1}\right)$ differentiates between uninfected nails and nails infected with dermatophytes, non-dermatophytes and yeasts. Model $2\left(\mathbf{M}_{2}\right)$, model $3\left(\mathbf{M}_{3}\right)$ and model $4\left(\mathbf{M}_{4}\right)$ discriminate between nails infected with the different dermatophyte, non-dermatophyte and yeast species, respectively. Data represent the mean of three independent training and test sets.

\begin{tabular}{|c|c|c|c|c|c|c|}
\hline Causative organisms & Samples & Training set (PCs) & Test set & Accuracy (\%) & Misclassified samples & Model \\
\hline Uninfected & 200 & $132(1)$ & 68 & 99.6 & 0.3 (dermatophytes) & $\mathrm{M}_{1}$ \\
\hline \multirow[t]{2}{*}{ Dermatophytes } & 238 & $156(5-6)$ & 82 & 95.9 & 1.7 (non-dermatophytes) & $\mathrm{M}_{1}$ \\
\hline & & & & & 1.7 (yeasts) & \\
\hline \multirow[t]{2}{*}{ T. rubrum } & 81 & $54(5-6)$ & 27 & 96.7 & $\begin{array}{l}0.3 \text { (T. violaceum }) \\
0.3(\text { M. canis })\end{array}$ & $\mathrm{M}_{2}$ \\
\hline & & & & & 0.3 (T. interdigitale $)$ & \\
\hline T. mentagrophytes & 19 & $12.7(1)$ & 6.3 & 73.0 & 1.7 (T. rubrum) & $\mathrm{M}_{2}$ \\
\hline \multirow[t]{2}{*}{ T. soudanense } & 19 & $12.7(1)$ & 6.3 & 84.1 & 0.3 (T. tonsurans) & $\mathrm{M}_{2}$ \\
\hline & & & & & 0.7 (T. violaceum) & \\
\hline M. persicolor & 19 & $12(1)$ & 7 & 95.7 & 0.3 (T. tonsurans) & $\mathrm{M}_{2}$ \\
\hline \multirow[t]{4}{*}{ T. tonsurans } & 19 & $11(2-3)$ & 8 & 85.0 & 0.3 (T. soudanense $)$ & $\mathrm{M}_{2}$ \\
\hline & & & & & $0.3($ T. violaceum $)$ & \\
\hline & & & & & 0.3 (M. canis) & \\
\hline & & & & & $0.3(T$. interdigitale $)$ & \\
\hline T. violaceum & 19 & $12(1-2)$ & 7 & 95.7 & 0.3 (T. rubrum) & $\mathrm{M}_{2}$ \\
\hline A. benhamiae & 22 & $14(1-2)$ & 8 & 91.3 & 0.7 (T. interdigitale $)$ & $\mathrm{M}_{2}$ \\
\hline M. canis & 22 & $14.7(2-4)$ & 7.3 & 95.9 & 0.3 (T. interdigitale $)$ & $\mathrm{M}_{2}$ \\
\hline T. interdigitale & 18 & $12.3(2-3)$ & 5.7 & 82.5 & 0.7 (T. rubrum) & $\mathrm{M}_{2}$ \\
\hline 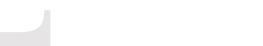 & & & & & 0.3 (M. canis) & \\
\hline Total & 238 & 155.4 & 82.6 & 91.0 & 7.4 & $\mathrm{M}_{2}$ \\
\hline Non-dermatophytes & 134 & $88(2-3)$ & 46 & 97.8 & 1 (dermatophytes) & $\mathrm{M}_{1}$ \\
\hline Acremonium & 39 & $26.7(1-4)$ & 12.3 & 97.6 & 0.3 (Fusarium) & $\mathrm{M}_{3}$ \\
\hline Fusarium & 37 & $24.7(1-4)$ & 12.3 & 100 & 0 & $\mathrm{M}_{3}$ \\
\hline S. brevicaulis & 20 & $13.3(1-3)$ & 6.7 & 89.6 & 0.7 (Fusarium) & $\mathrm{M}_{3}$ \\
\hline Penicillium & 38 & $25.7(1)$ & 12.3 & 100 & 0 & $\mathrm{M}_{3}$ \\
\hline Total & 134 & 87 & 43.6 & 97.7 & 1.0 & $\mathrm{M}_{3}$ \\
\hline Yeasts & 62 & $41(3-5)$ & 21 & 90.5 & 1.3 (dermatophytes) & $\mathrm{M}_{1}$ \\
\hline & & & & & 0.7 (non-dermatophytes) & \\
\hline C. albicans & 19 & $12(1)$ & 7 & 100 & 0 & $\mathrm{M}_{4}$ \\
\hline C. krusei & 20 & $13(1)$ & 7 & 100 & 0 & $\mathrm{M}_{4}$ \\
\hline
\end{tabular}




\begin{tabular}{|c|c|c|c|c|c|c|}
\hline C. parapsilosis & 23 & $15(1-3)$ & 8 & 96.3 & 0.3 (C. albicans) & $\mathrm{M}_{4}$ \\
\hline Total & 62 & 40 & 22 & 98.6 & 0.3 & $\mathrm{M}_{4}$ \\
\hline Total & 634 & 420 & 214 & 96.9 & 6.7 samples & $\mathrm{M}_{1}$ \\
\hline
\end{tabular}

Abbreviations: PCs, number of principal components used to model microorganism classes. 
Table 3: Demographic characteristics, comorbidities and medication use of subjects enrolled in the

in vivo pilot model.

Characteristics

Demographic

Age (year), median $\pm \mathrm{SD}$

Men/women, $\mathrm{n} / \mathrm{n}$

\section{Comorbidity}

Asthma, $\mathrm{n}$

Chronic obstructive pulmonary disease, $\mathrm{n}$

Colitis ulcerosa, $\mathrm{n}$

Depressive disorder, $\mathrm{n}$

Diabetes, $\mathrm{n}$

Dyslipidemia, $\mathrm{n}$

Emphysema, $\mathrm{n}$

Heart rhythm disorder, $\mathrm{n}$

Hidradenitis suppurativa, $\mathrm{n}$

Hypertension, $\mathrm{n}$

Hypogonadotropic hypogonadism, $n$

Hypothyroidism, n

Ischemic heart disease, $\mathrm{n}$

Malignancy, n

Migraine, $\mathrm{n}$

Obesity/metabolic syndrome, $\mathrm{n}$

Obstructive sleep apnoea syndrome, $\mathrm{n}$

Psoriasis, $n$
$59.5 \pm 6.2$

$12 / 6$

$9 / 3$

$\begin{array}{ll}0 & 1 \\ 0 & 1 \\ 0 & 1 \\ 0 & 1 \\ 1 & 1 \\ 3 & 1 \\ 0 & 2 \\ 0 & 1 \\ 1 & 2 \\ 2 & 1 \\ 0 & 2 \\ 2 & 1 \\ 0 & 1 \\ 0 & 1 \\ 0 & 1 \\ 1 & 1 \\ 0 & 1 \\ 1 & 1 \\ & 4\end{array}$

Medication use (systemic)

ACE-inhibitors, $n$

Acetylsalicylic acid, $\mathrm{n}$

Allopurinol, $\mathrm{n}$

Analgetics, $n$

Antibiotics, $\mathrm{n}$

Benzodiazepines, $\mathrm{n}$

Beta-blockers, $\mathrm{n}$

Bisphosphonates, $\mathrm{n}$

Calcium antagonists, $\mathrm{n}$

Diuretics, $\mathrm{n}$

Fibrates, n

Leflunomide, $\mathrm{n}$

Levothyroxine, $\mathrm{n}$

Metformine, $\mathrm{n}$

Methotrexate, $\mathrm{n}$

Nonsteroidal anti-inflammatory drugs, $\mathrm{n}$

Control group

Onychomycosis group

$(n=18)$

$(n=12)$

Proton pump inhibitors, $\mathrm{n}$

Rivaroxaban, $\mathrm{n}$

Statins, $\mathrm{n}$

Testosteron, $\mathrm{n}$

TNF-alpha inhibitors, $\mathrm{n}$

\begin{tabular}{|c|c|}
\hline 1 & 3 \\
\hline 1 & 3 \\
\hline 0 & 1 \\
\hline 0 & 1 \\
\hline 0 & 2 \\
\hline 0 & 2 \\
\hline 2 & 5 \\
\hline 0 & 1 \\
\hline 0 & 1 \\
\hline 1 & 3 \\
\hline 0 & 1 \\
\hline 0 & 1 \\
\hline 2 & 1 \\
\hline 0 & 2 \\
\hline 0 & 1 \\
\hline 0 & 3 \\
\hline 0 & 4 \\
\hline 0 & 1 \\
\hline 3 & 2 \\
\hline 0 & 1 \\
\hline 0 & 1 \\
\hline
\end{tabular}

Abbreviations: n, number; SD, standard deviation.

This article is protected by copyright. All rights reserved. 


\section{FIGURES}

Figure 1. Raw absorbance spectra. Typical molecular assignments of a biological infrared spectrum are indicated, $v$ : stretching vibrations, $\delta$ : bending vibrations, $s:$ symmetric vibrations, as: asymmetric vibrations. (A) Mean raw spectra of cultured Trichophyton rubrum (T. rubrum) strains (red line), intact control toenails (green line) and intact toenails infected ex vivo with $T$. rubrum (blue line). Notice the strong absorbance of carbohydrates in the $1200-900 \mathrm{~cm}^{-1}$ region due to ring C-O-C vibrations in case of cultured strains. Since no drying procedure was applied to cultured strains, fundamental $\mathrm{O}-\mathrm{H}$ stretching vibrations induce a strong absorbance at $3314 \mathrm{~cm}^{-1}$. (B) Mean raw spectra of powdered control toenails (green line) and powdered toenails infected in vivo with T. rubrum (blue line). Raw absorbance spectra show a high level of similarity between control and infected toenails. To draw vital information from the spectral data, data pretreatment (e.g. derivatization) and appropriate chemometric techniques are necessary.

Figure 2. Infrared spectra of cultured fungi. (A) Dermatophytes. Mean spectral differences in the second derivative of the range between 1692-1606 (amide I band containing mainly $\mathrm{C}=\mathrm{O}$ stretching vibrations of protein peptide bonds) and 1044-1004 $\mathrm{cm}^{-1}$ (part of the carbohydrate region) for dermatophytes $(n=20)$. The corresponding dendrogram, obtained from hierarchical cluster analysis (HCA), shows two main clusters representing Microsporum spp. (cluster 1) and Trichophyton spp. (cluster 2). (B) Non-dermatophytes/yeasts. ( $n=8$ and $n=4$, respectively). Mean spectral differences in second derivative of the spectral range between $973-937 \mathrm{~cm}^{-1}$ (part of the carbohydrate region) with corresponding dendrogram showing clustering of all Candida species and non-dermatophyte fungi (Fusarium spp., Penicillium spp. and Acremonium spp.), with exception of Scopulariopsis brevicaulis that forms a separate cluster. A second-order derivative spectrum is a plot of the curvature of the absorption (A) spectrum against wavelength $(\lambda)$ obtained by measuring alterations in the rate of change of absorbance $\left(\mathrm{d}^{2} \mathrm{~A} / \mathrm{d} \lambda^{2}\right)$. For HCA, the vertical axis indicates the loss in within cluster similarity (i.e. the variance increase), when clusters are merged. The scale on the vertical axis is rather arbitrary.

This article is protected by copyright. All rights reserved. 
Figure 3. Ex vivo nail model. (A) Mean spectral differences in the second derivative from 1200 to 900 $\mathrm{cm}^{-1}$ (carbohydrate region) between uninfected $(\mathrm{n}=200$, green line $)$ and ex vivo infected nails $(\mathrm{n}=434$, blue line). A second-order derivative spectrum is a plot of the curvature of the absorption (A) spectrum against wavelength $(\lambda)$ obtained by measuring alterations in the rate of change of absorbance $\left(\mathrm{d}^{2} \mathrm{~A} / \mathrm{d} \lambda^{2}\right)$. (B) Score plot showing clear clustering of uninfected (green dots) and ex vivo infected nails (blue dots), based on principal component analysis (PCA) in the $1200-900 \mathrm{~cm}^{-1}$ spectral range.

Figure 4. In vivo nail model. (A) First derivative of the mean spectra from uninfected (green line) and infected nails (blue line) with close-ups on spectral differences in three regions: $3000-2811 \mathrm{~cm}^{-1}$ (region assigned to (a)symmetric $\mathrm{CH}_{2}$ and $\mathrm{CH}_{3}$ stretching vibrations of lipids), $1676-1553 \mathrm{~cm}^{-1}$ (amide I band) and 1043-950 $\mathrm{cm}^{-1}$ (part of the carbohydrate region). A first-order derivative spectrum is a plot of the gradient of the absorption curve (rate of change of absorbance with wavelength, $\mathrm{dA} / \mathrm{d} \lambda$ ) against wavelength. (B) Score plot showing clear clustering of uninfected (healthy nails and nails negative for both Calcofluor white $(\mathrm{CW})$ stain and culture, green dots) and infected nails (positive $\mathrm{CW}$ stain and/or culture, blue dots) based on principal component analysis (PCA) on these regions. (C) Dendrogram, obtained from hierarchical cluster analysis (HCA), showing 2 main clusters representing uninfected (green cluster) and infected nails (blue cluster). For HCA, the vertical axis indicates the loss in within cluster similarity (i.e. the variance increase), when clusters are merged. The scale on the vertical axis is rather arbitrary.

This article is protected by copyright. All rights reserved. 

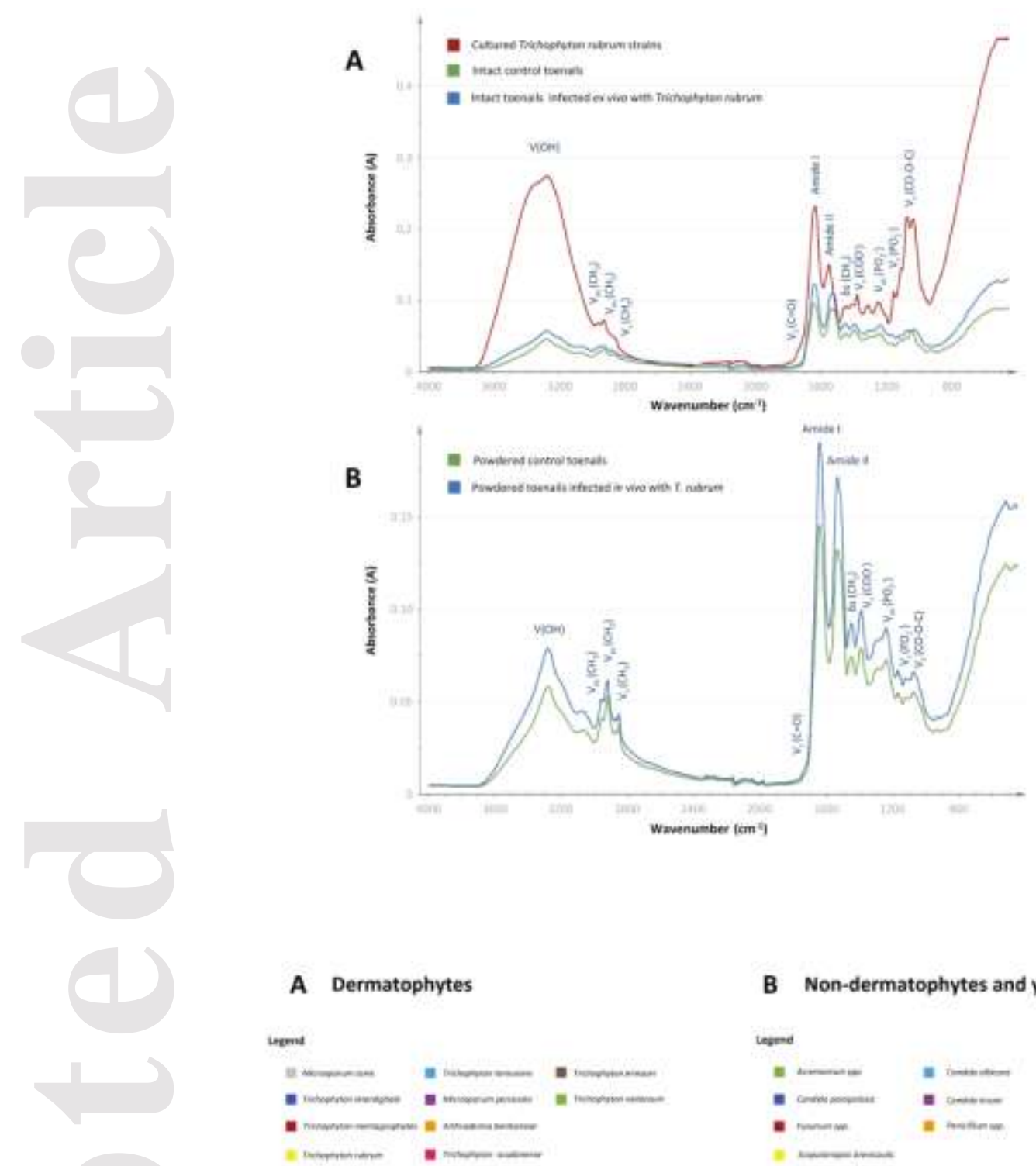

B Non-dermatophytes and yeasts
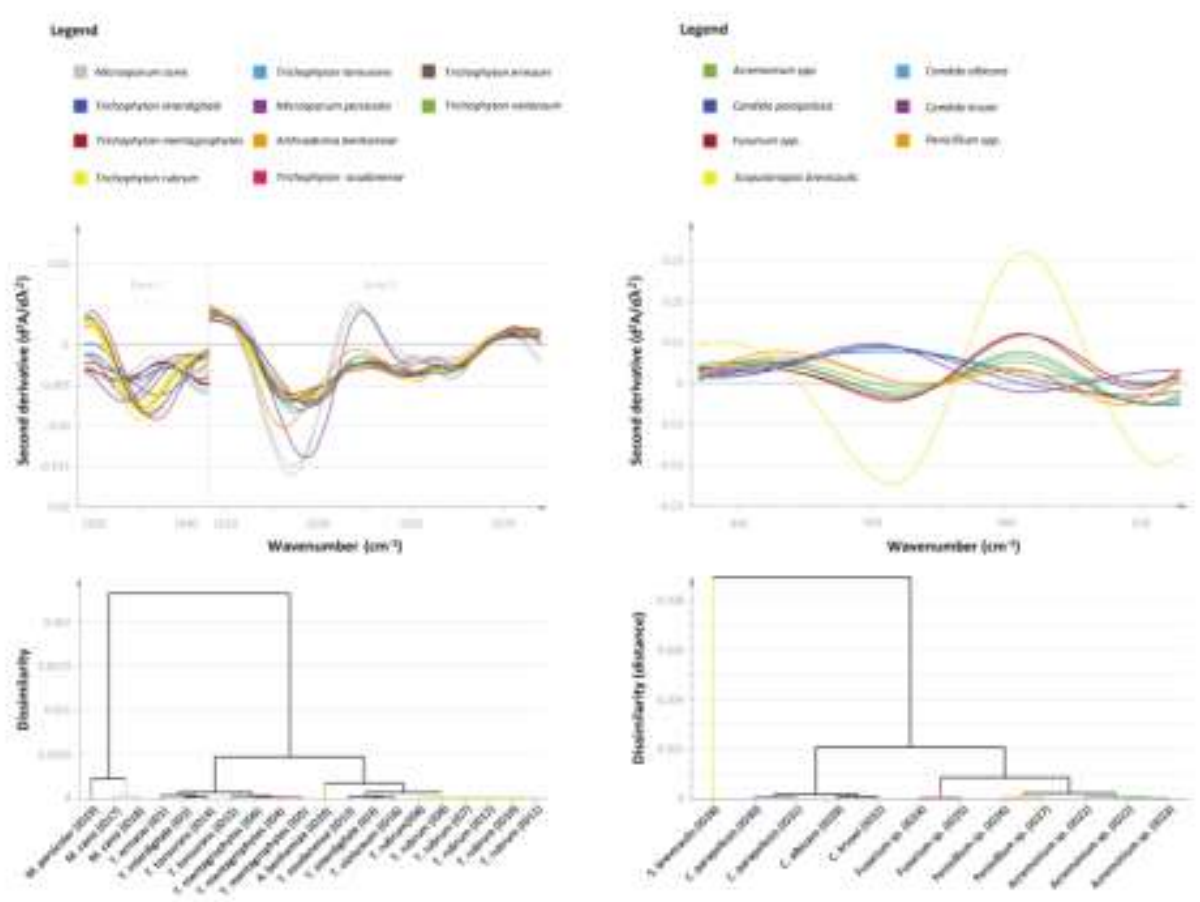

This article is protected by copyright. All rights reserved. 


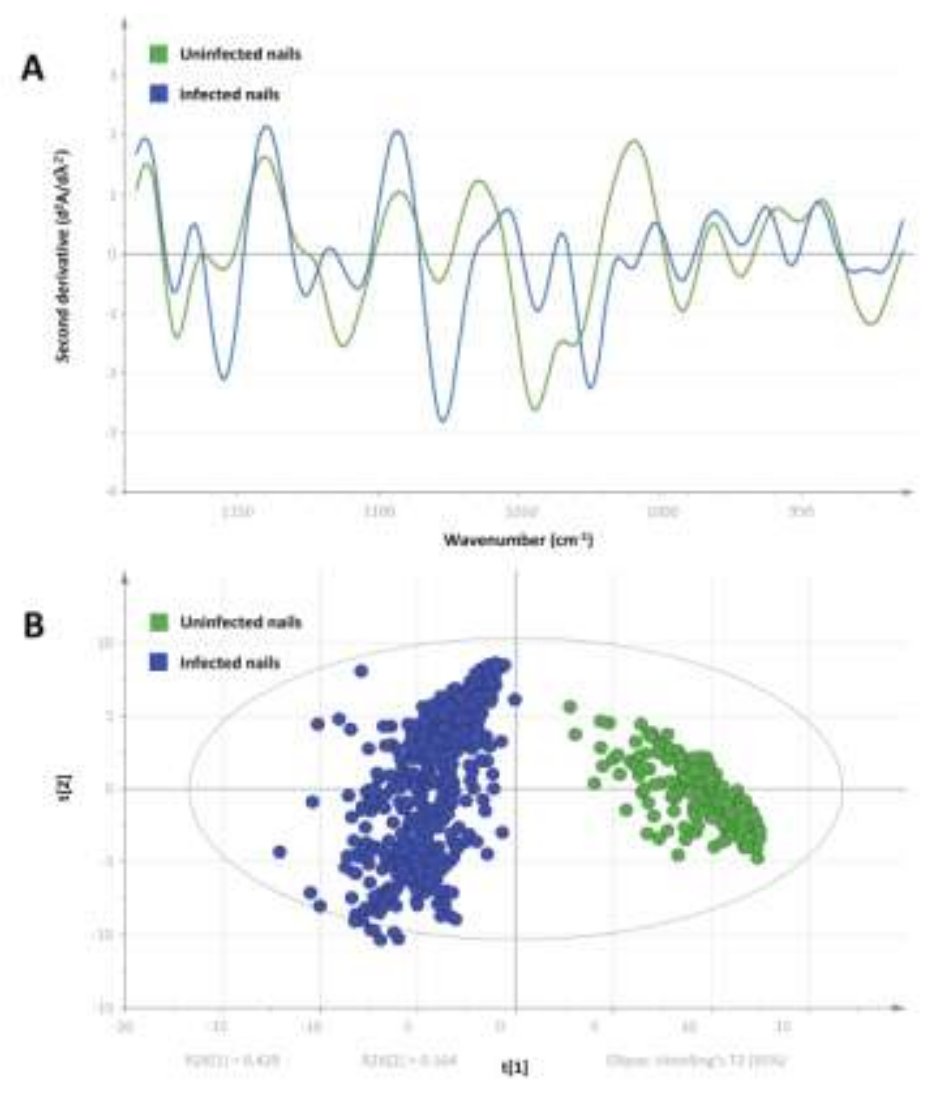

This article is protected by copyright. All rights reserved. 

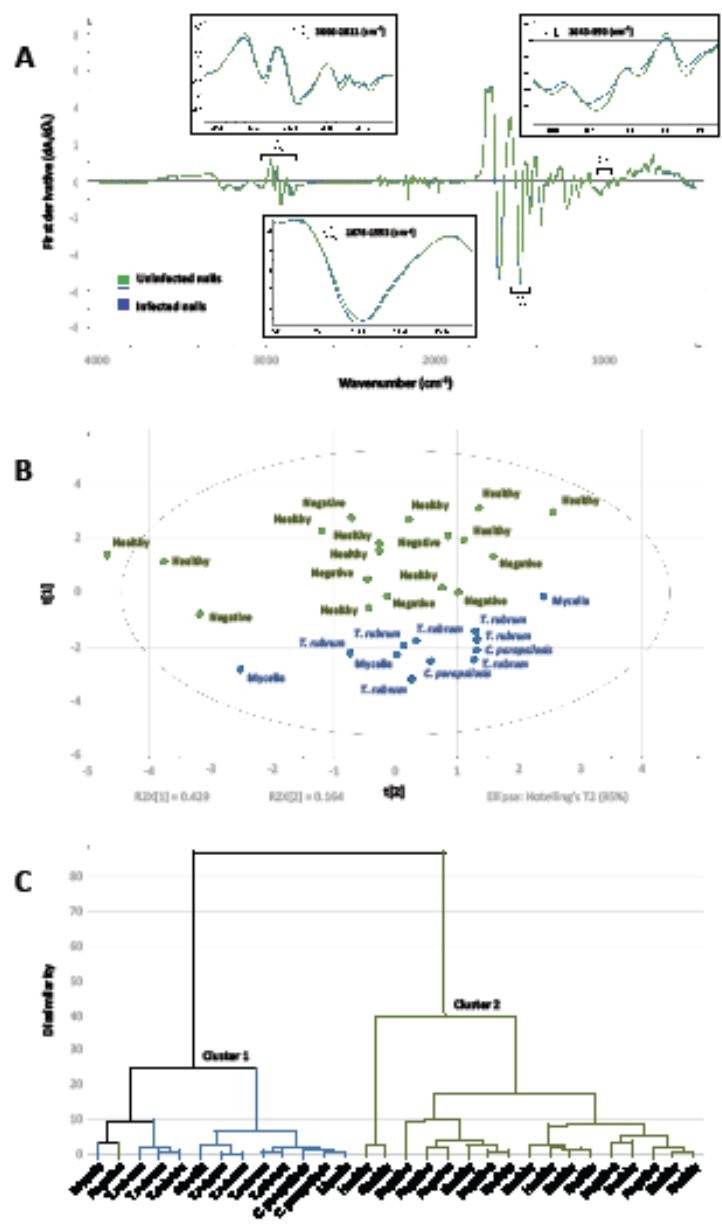

This article is protected by copyright. All rights reserved. 